\title{
MPLS Traffic Engineering in Satellite Networks
}

\author{
Arjan Durresi \\ Department of Computer Science, Louisiana State University \\ Baton Rouge, Louisiana, USA, Email: durresi@csc.lsu.edu \\ Mimoza Durresi, Leonard Barolli \\ Department of Information and Communication Engineering \\ Fukuoka Institute of Technology, Fukuoka, Japan \\ Fatos Xhafa \\ Department of Languages and Informatics Systems \\ Polytechnic University of Catalonia, Barcelona, Spain
}

\begin{abstract}
Broadband satellite constellation networks will be required to carry all types of IP traffic, real time interactive traffic as well as non-real time traffic, warranting the need for appropriate QoS for these different traffic flows. In this paper we investigate the need for MPLS traffic engineering in GEO/MEO/LEO satellite networks to address QoS issues. We compare the service received by TCP and UDP flows when they share a link and when they are routed on explicit MPLS traffic trunks. Since MPLS traffic trunks allow non-shortest path links also to be used, the total network throughput goes up with proper traffic engineering. If UDP and TCP flows are mixed in a trunk, TCP flows receive reduced service as the UDP flows increase their rates. Also, we found that with MPLS traffic engineering we can protect real time traffic and VoIP traffic from packet loss and excessive jitter by separating them from other congestion unresponsive flows.
\end{abstract}

\section{Introduction}

With the continuous increase in IP traffic it has become necessary to utilize satellite networks for transport of internet traffic including real-time multimedia applications. Satellite networking uses from simple bent-pipe routing for GEO satellite networks to on-board switching capabilities in LEO/MEO broadband satellite constellations $[12,1,7]$. GEO satellites orbiting in geo-stationary orbits acted as transparent bent pipes between ground stations with no routing functionality. Because of their high altitude orbits $(36000 \mathrm{Km})$ propagation delay is large, conse- quently making them unsuitable for real-time applications. All these issues motivated the deployment of low-earth orbit(LEO) satellites which orbit the Earth at a height of just 500 to 1,000 miles, which in turn necessitates the use of multiple satellites which constantly orbit around the earth in fixed planes to provide constant service to any area. The LEO constellation can be viewed as a mobile network with fixed users and mobile nodes. The low altitude orbit makes them capable of providing smaller, more energy-efficient spot beams, and delivers latency potentially equal to (or better than) transcontinental fiber optic cable. Frequency reuse is also an important advantage considering the limited and costly frequency spectrum while increasing the system capacity.

With the advent of multiple spot beams, inter-satellite links (ISLs) between satellites and on board switching and processing capabilities, these constellation of low-earth orbit(LEO/MEO) satellites along with their terrestrial gateway servers form Autonomous systems(AS). Most companies that want to provide satellite-based Internet access are deploying Low Earth Orbit (LEO) satellite constellations e.g. Iridium, Teledesic. One of the distinct advantages of LEO satellite networks over GEO networks is the reduction in propagation delay making them an attractive option for routing real time traffic.

The rest of the paper is organized as follows, Section 2 discusses MPLS and the need for traffic engineering in satellite networks. Section 3 analyzes the simulation results and Section 4 concludes. 


\section{MPLS in Satellite Networks}

\subsection{MPLS Overview}

MPLS stands for "Multiprotocol Label Switching". It's a layer 3 switching technology aimed at greatly improving the packet forwarding performance of the backbone routers in the Internet or other large networks. The basic idea is to forward the packets based on a short, fixed length identifier termed as a 'label', instead of the network-layer address with variable length match. The labels are assigned to the packets at the ingress node of an MPLS domain. Inside the MPLS domain, the labels attached to packets are used to make forwarding decisions. Thus, MPLS uses indexing instead of a longest address match as in conventional IP routing. The labels are finally popped out from the packets when they leave the MPLS domain at the egress nodes. By doing this, the efficiency of packet forwarding is greatly improved. Routers which support MPLS are known as "Label Switching Routers", or "LSRs" [4]

Although the original idea behind the development of MPLS was to facilitate fast packet switching, currently its main goal is to support traffic engineering and provide quality of service. The goal of traffic engineering is to facilitate efficient and reliable network operations, and at the same time optimize the utilization of network resources $[8,3,5,11]$. Most current network routing protocols are based on the shortest path algorithm, which implies that there is only one path between a given source and destination end system.

In contrast, MPLS supports explicit routing, which can be used to optimize the utilization of network resources and enhance traffic oriented performance characteristics. For example, multiple paths can be used simultaneously to improve performance from a given source to a destination. MPLS provides explicit routing without requiring each IP packet to carry the explicit route, which makes traffic engineering easier. Another advantage is that using label switching, packets of different flows can be labeled differently and thus received different forwarding (and hence different quality of service).

A Label Switched Path (LSP) is referred to as a path from the ingress node to the egress node of an MPLS domain followed by packets with the same label. A traffic trunk is an aggregation of traffic flows of the same class, which are placed inside an LSP, as shown in Fig. 1. Therefore, all packets on a traffic trunk have the same label and the same 3-bit class of service field in the MPLS header. Traffic trunks are routable objects. These trunks can be established either statically or dynamically ( on demand ) between any two nodes in an MPLS domain.

A trunk can carry any aggregate of micro-flows, where each micro-flow consists of packets belonging to a single
TCP or UDP flow. In general, trunks are expected to carry several such micro-flows of different transport types. However, as shown in this analysis, mixing different transport types can cause performance problems such as starvation and unfairness for certain traffic flows $[10,9,5]$.

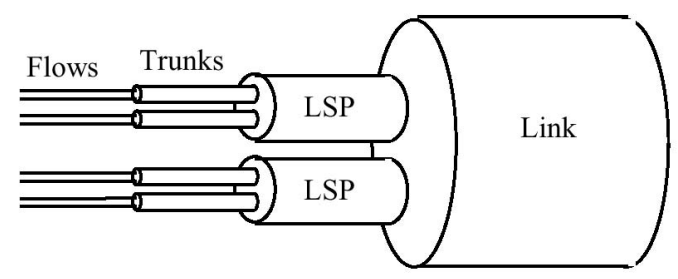

\section{Figure 1. Illustrations of the relationships be- tween the various MPLS concepts.}

\subsection{Need for MPLS in Satellite Networks}

In general, satellite bandwidth is scarcer and more expensive than terrestrial. That's mean QOS techniques are more needed in satellite environments than in terrestrial ones. MPLS is a good candidate to apply QOS in general and traffic engineering in particular in satellite networks. Since MPLS operates independently of layer 3 and will use IP routing methods, standard IP QoS can be enforced during the LSP setup process. LSPs with specific bandwidth requirements, delay bounds can be setup using constraintbased routing and have labels associated with them. Consequently appropriate traffic can be routed along their desired QoS path.

\subsection{Traffic Engineering Requirements in Satellite Networks}

The need for QoS in satellite networks is fueled by several reasons. With an explosion of network traffic in terms of users and applications, ISPs want to offer different levels of service based on business priorities of the users or applications. With applications varying from real time interactive traffic (e.g. VoIP), real time non-interactive traffic(e.g. streaming video) to non-real time traffic(e.g. web traffic) it is necessary to differentiate in the levels of service provided. High speed networks should be able to support different degrees of Quality of Service (QoS) to different applications. For example, real-time traffic generated by multimedia applications has radically different requirements than best-effort traffic. First, real-time applications require tight bounds on transfer delay (in the order of hundreds of milliseconds). Second, the loss probability of network packets belonging to multimedia applications must be 


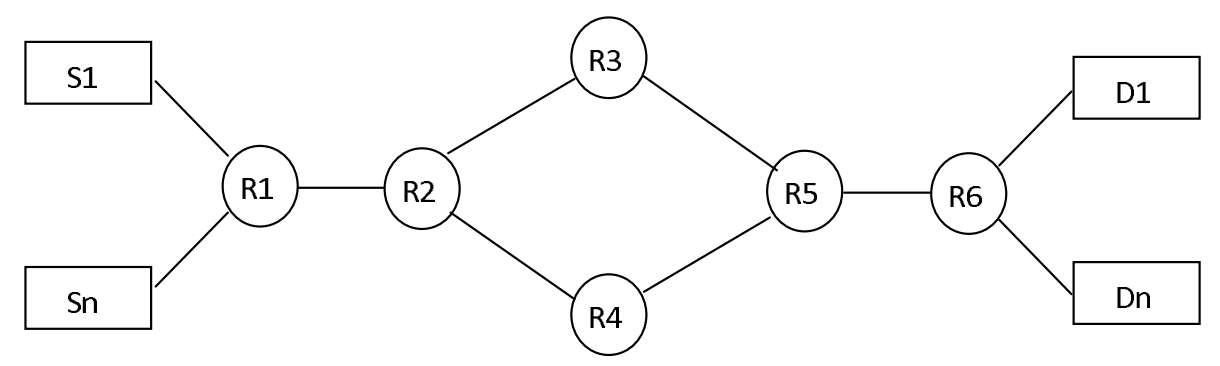

Figure 2. Network Topology.

very small (varying from $10^{-12}$ to $10^{-13}$ depending on the kind of application).

Real-time applications such as VoIP and streaming video, are susceptible to changes in the transmission characteristics of data networks. Voice over IP (VoIP) and real time traffic(VBR) are also susceptible to network behaviors, referred to as delay and jitter, which can degrade the voice application to the point of being unacceptable to the average user. So it becomes essential to separate such high priority traffic from non-real time traffic e.g. file transfer and route them of explicit paths which meet the desired QoS requirements.

\section{Network Configuration}

For analyzing the effect of using MPLS over satellite networks we use the ns-2 simulator [2].

In the simulations the network topology shown in Fig. 2 was used. Routers R1, R2 , R5 and R6 are terrestrial routers. Routers R3 and R4 are satellite ones. The uplink and downlink bandwidth from the terrestrial stations to the satellites is $5 \mathrm{Mb}$. The routers are MPLS capable. There are 3 flows. Source S1 sends UDP traffic to destination D1. Sources $\mathrm{S} 2$ and S3 send TCP traffic to destination D2 and D3, respectively (here $n=3$ ). The TCP sources are "infinite ftp" sources and send packets whenever its congestion window allows. The actual throughput are monitored at the destination nodes. We use a number of VoIP sources to represent real-time interactive traffic, two TCP sources as non-real time web traffic and a UDP source with variable bit rate as streaming video.

The parameters measured are throughput, delay, jitter and packet loss. We define the parameters as pertaining to our simulations:

Delay is the time taken from point-to-point in a network. Delay can be measured in either one-way or round-trip delay. To get a general measurement of one-way delay, measure round-trip delay and divide the result by two. VoIP typically tolerates delays up to $150 \mathrm{~ms}$ before the quality of the call is unacceptable. In our simulations we measure one-way mean delay.
Jitter is the variation in delay over time from point-topoint. If the delay of transmissions varies too widely in a VoIP call, the call quality is greatly degraded. The amount of jitter tolerable on the network is affected by the depth of the jitter buffer on the network equipment in the voice path. The more jitter buffer available, the more the network can reduce the effects of jitter. We measure the jitter as the standard deviation of the one-way delay.

Packet loss is losing packets along the data path, which severely degrades the voice application.

\section{LEO Results}

\subsection{Throughput Analysis}

The first case we analyzed is the overall throughput performance of the network with and without MPLS. We generate VoIP and two TCP sources for this purpose.

\section{Case 1: No trunks, No MPLS}

According to the current satellite routing strategy implemented in the ns-2 simulator, which basically routes through the shortest path available. All three flows use the same route. The TCP flows being inherently congestion responsive are cut down by the VoIP flow.

Fig. 3 illustrates the relation between the VoIP rate and the throughput of the three flows.

Case 2: Two separate trunks using Label Switched Paths

Next we analyze the same traffic but in this case with MPLS we explicitly route TCP2 on a separate LSP, while the VoIP and TCP1 share another LSP. Fig. 4 illustrates the relation between the throughput and the corresponding VoIP rate.

Since the TCP2 flow is routed explicitly on a $5 \mathrm{Mbps}$ link, increase in the VoIP rate has no effect on TCP2 throughput. TCP1 though which shares the same LSP with VoIP traffic suffers as the VoIP rate is increased. Also we note that the overall throughput of the network has gone above 9 Mbps. 


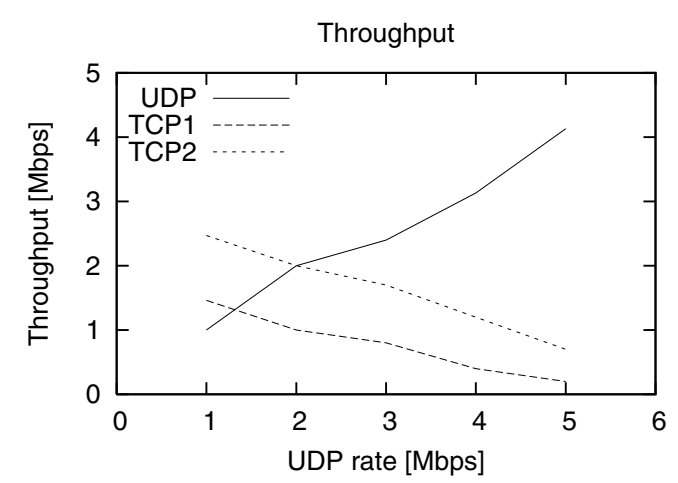

Figure 3. UDP and TCP throughput without the use of MPLS.

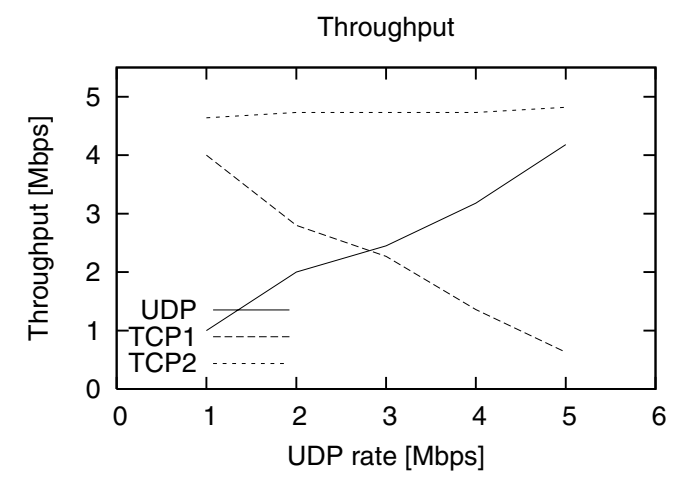

Figure 4. UDP and TCP throughput using of MPLS.

\subsection{Jitter and Packet Loss Analysis}

Since the TCP2 flow is routed explicitly on a 5Mbps link, increase in the VoIP rate has no effect on TCP2 throughput. TCP1 though which shares the same LSP with VoIP traffic suffers as the VoIP rate is increased. Also we note that the overall throughput of the network has gone above 9 Mbps.

Jitter is a major concern for real-time traffic and to maintain necessary QoS it has be kept as low as possible. To analyze jitter we used the same simulation scenario as for the throughput analysis.

Case 1: No trunks, No MPLS

Jitter is a major concern for real-time traffic and to maintain necessary QoS it has be kept as low as possible. To analyze jitter we used the same simulation scenario as for the throughput analysis.

Since the main purpose of our analysis is to ascertain the need for traffic engineering in satellite networks and not determining the exact values of jitter, the simulation time is for a very small period, hence the values of jitter may increase for larger periods e.g. one whole day. Typical jitter values range anywhere from $23 \mathrm{~ms}$ to $60 \mathrm{~ms}$ or even more than $100 \mathrm{~ms}$ for distances more than $15,000 \mathrm{~km} \mathrm{[6].}$

Jitter analysis though showed something interesting, the more the number of VoIP sources, the higher the mean latency, but jitter kept reducing as shown in the results of Tab. 2. The explanation for this is that with higher bandwidth of VoIP traffic, the TCP flow was reduced because of its congestion responsive nature, therefore having less effect on the queue and keeping jitter low, but when VoIP traffic was less, due to increased TCP traffic, the jitter value increased.

Table 1. Packet Loss, Delay and Jitter. Results without MPLS

\begin{tabular}{ccc}
\hline Number of VoIP sources & 400 & 520 \\
\hline \hline VoIP bandwidth (Mbps) & 3.2 & 4.2 \\
VoIP packet Loss (\%) & 3.26 & 10.77 \\
Mean delay (ms) & 52.38 & 56.23 \\
Jitter (ms) & 13.82 & 9.83 \\
\hline
\end{tabular}

Case 2: With MPLS

The VoIP traffic is explicitly routed along a path different from that of the two TCP flows. One of the drawbacks of this is that there is a possibility that the mean delay of this path might be more than the shortest path.

With the VoIP traffic separated from the TCP flows both the packet loss and jitter values have come within acceptable values for VoIP traffic.

Table 2. Packet Loss, Delay and Jitter. Results with MPLS

\begin{tabular}{ccc}
\hline Number of VoIP sources & 400 & 520 \\
\hline \hline VoIP bandwidth (Mbps) & 3.2 & 4.2 \\
VoIP packet Loss (\%) & 0.37 & 0.5 \\
Mean delay (ms) & 35.05 & 36.24 \\
Jitter (ms) & 0.33 & 0.86 \\
\hline
\end{tabular}

\section{MEO Results}

The experiments are similar to LEO case, except that RTT is $240 \mathrm{~ms}$.

Case 1: No trunks, No MPLS

As shown in Fig. 5, TCP throughput will suffer from the competition with UDP throughput.

Case 2: With MPLS trunk

Next we analyze the same traffic but in this case with MPLS we explicitly route TCP1 on a separate LSP, while the VoIP and TCP2 share another LSP. As shown in Fig. 6, TCP1 throughput is not affected by UDP traffic. 


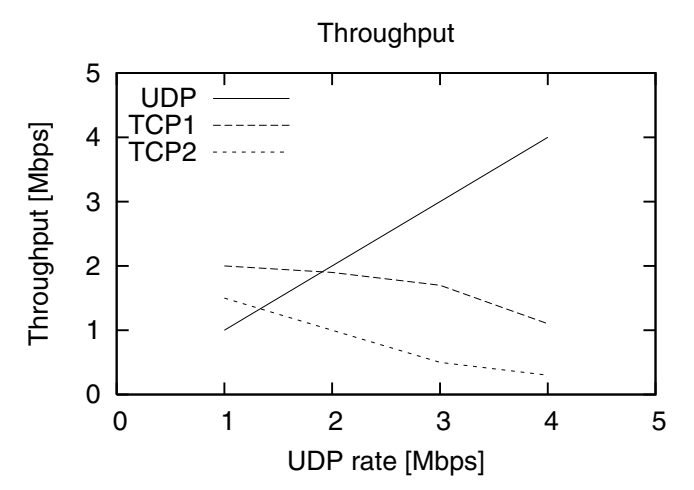

Figure 5. UDP and TCP throughput without the use of MPLS.

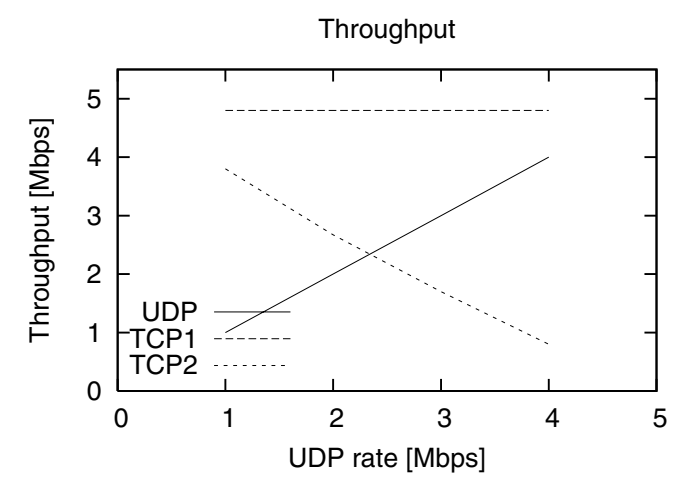

Figure 6. UDP and TCP throughput using of MPLS.

\section{GEO Results}

The experiments are similar to LEO case, except that RTT is $520 \mathrm{~ms}$.

Case 1: No Trunks, No MPLS

As shown in Fig. 7, TCP throughput will suffer from the competition with UDP throughput.

Case 2: With MPLS trunk

TCP1 is explicitly routed on a separate $5 \mathrm{Mbps}$ MPLS trunk. TCP2 and UDP are together on the same $5 \mathrm{Mbps}$ trunk. As shown in Fig. 8, TCP1 throughput is not affected by UDP traffic.

\section{Conclusions}

We conclude from the above results that separating real time (congestion unresponsive) flows which are sensitive to end-to-end latency and jitter from congestion-responsive flows e.g. TCP is a necessary QoS requirement which can be achieved through MPLS traffic engineering. There is

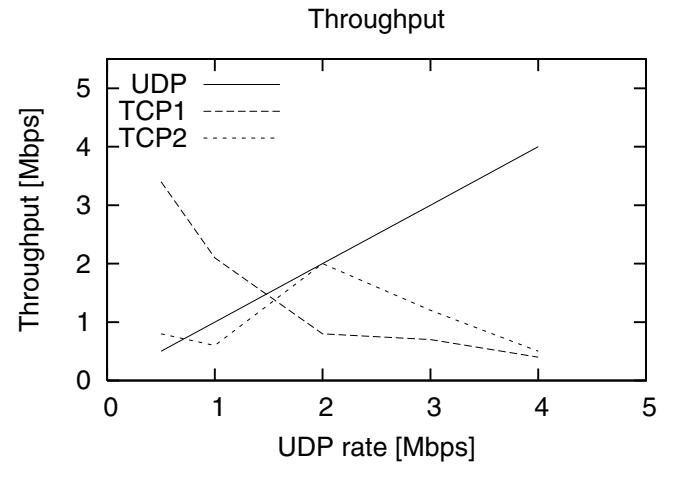

Figure 7. UDP and TCP throughput without the use of MPLS.

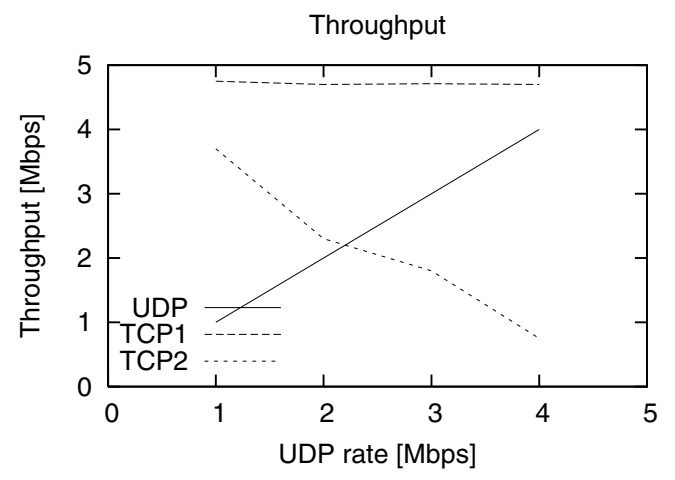

Figure 8. UDP and TCP throughput using of MPLS.

a significant improvement in the overall network throughput. Therefore different flows with different QoS requirements should be in different trunks to guarantee the necessary QoS. MPLS could also provide the best support for real time traffic over IP networks.

\section{References}

[1] Iridium Satellite. http://www.iridium.com/, January 2007.

[2] The Network Simulator - ns-2. http://nsnam.isi.edu/nsnam/index.php/User_Information, January 2007.

[3] D. O. Awduche, J. Malcolm, and M. O. amd J. McManus. Requirements for Traffic Engineering Over MPLS. draftietf-mpls-traffic-eng-00.txt, October 1998.

[4] P. Bhaniramka, W. Sun, and R. Jain. Quality of service using traffic engineering over mpls: An analysis. In Proc. of $L C N$ 2000, pages 238-241, Tampa, Florida USA, Nov. 2000.

[5] S. Floyd and V. Jacobson. Link-sharing and resource management models for packet networks,. IEEE/ACM Transactions on Networking, 3(4):365-386, 1995. 
[6] T. R. Henderson and R. H. Katz. Network simulation for leo satellite networks. In Proc. 18th AIAA International Communications Satellite Systems Conference (ICSSC), Oakland, California, April 2000.

[7] A. Jukan, H. N. Nguen, and H. R. V. As. An approach to qos based routing in leo satellite networks. In Proc. International Conference on Communication Technology WCC ICCT 2000, 2000.

[8] T. Li and Y. Rekhter. A Provider Architecture for Differentiated Services and Traffic Engineering (PASTE). In IETF, editor, RFC 2430, October 1998.

[9] E. C. Rosen and Y. Rkhter. MPLS Label Stack Encoding. draft-ietf-mpls-label-encaps-03.txt, September 1998.

[10] E. C. Rosen, A. Vishwanathan, and R. Callon. Multiprotocol Label Switching Architecture. draft-ietf-mpls-arch-04.txt, February 1999.

[11] L. Wischhof and J. Lockwood. Packet scheduling for linksharing and quality of service support in wireless local area networks. Technical Report WUCS-01-35, Applied Research Laboratory, Washington University in St. Louis, Nov. 2001.

[12] L. Wood, A. Clerget, I. Andrikopoulos, G. Pavlou, and W. Dabbous. Ip routing issues in satellite constellation networks,. International Journal of Satellite Communications, 19(1):69-92, January/February 2001. 